\title{
REPRESENTATIONS OF THE ORLICZ FIGÀ-TALAMANCA HERZ ALGEBRAS AND SPECTRAL SUBSPACES
}

\author{
RATTAN LAL AND N. SHRAVAN KUMAR
}

\begin{abstract}
Let $G$ be a locally compact group. In this note, we characterise nondegenerate *-representations of $A_{\Phi}(G)$ and $B_{\Phi}(G)$. We also study spectral subspaces associated to a non-degenerate Banach space representation of $A_{\Phi}(G)$.
\end{abstract}

\section{INTRODUCTION}

Let $G$ be a locally compact group. It is well known that there is a one to one correspondence between the unitary representations of $G$ and the non-degenerate $*$-representations of $L^{1}(G)$ [5, p. 73]. Similarly, if $X$ is any locally compact Hausdorff space, then there is a one to one correspondence between the cyclic $*$-representations of $C_{0}(X)$ and positive bounded Borel measures on $X$ [8, p. 486]. The corresponding result for the Fourier algebra $A(G)$ of a locally compact group is due to Lau and Losert [10]. For more on the Fourier algebra see [4, 9]. Recently, Guex [11] extended the result of Lau and Losert to Figà-Talamanca Herz algebras. We refer the readers to [2] for more on Figà-Talamanca Herz algebras.

In [14], the authors have introduced and studied the $L^{\Phi}$-versions of the Figà-Talamanca Herz algebras. Here $L^{\Phi}$ denotes the Orlicz space corresponding to the Young function $\Phi$. The space $A_{\Phi}(G)$ is defined as the space of all continuous functions $u$, where $u$ is of the form

$$
u=\sum_{n=1}^{\infty} f_{n} * \check{g_{n}}
$$

where $f_{n} \in L^{\Phi}(G), g_{n} \in L^{\Psi}(G),(\Phi, \Psi)$ is a pair of complementary Young functions satisfying the $\Delta_{2}$-condition and

$$
\sum_{n=1}^{\infty} N_{\Phi}\left(f_{n}\right)\left\|g_{n}\right\|_{\psi}<\infty
$$

It is shown in [14] that $A_{\Phi}(G)$ is a regular, tauberian, semisimple commutative Banach algebra with the Gelfand spectrum homeomorphic to $G$.

This paper has the modest aim of characterising the non-degenerate $*$-representations of $A_{\Phi}(G)$ in the spirit of [10]. This characterisation is given in Corollary 3.4. In Section 4 , we show that any non-degenerate $*$-representation of $A_{\Phi}(G)$ can be extended uniquely to a non-degenerate $*$-representation of $B_{\Phi}(G)$. In Section 5 , we provide an application to ergodic sequences.

Godement in his fundamental paper [6] on Wiener Tauberian theorems studied spectral subspaces associated to a certain Banach space representations. This result was extended to the Fourier algebra $A(G)$ by Parthasarathy and Prakash [12]. In Section 6, we also study spectral subspaces of $A_{\Phi}(G)$.

2010 Mathematics Subject Classification. Primary 43A15, 46J25; Secondary 22 D99.

Key words and phrases. Orlicz Figà-Talamanca Herz algebra, representation, spectral subspaces. 


\section{Preliminaries}

Let $\Phi: \mathbb{R} \rightarrow[0, \infty]$ be a convex function. Then $\Phi$ is called a Young function if it is symmetric and satisfies $\Phi(0)=0$ and $\lim _{x \rightarrow \infty} \Phi(x)=+\infty$. If $\Phi$ is any Young function, then define $\Psi$ as

$$
\Psi(y):=\sup \{x|y|-\Phi(x): x \geq 0\}, \quad y \in \mathbb{R} .
$$

Then $\Psi$ is also a Young function and is termed as the complementary function to $\Phi$. Further, the pair $(\Phi, \Psi)$ is called a complementary pair of Young functions.

Let $G$ be a locally compact group with a left Haar measure $d x$. We say that a Young function $\Phi$ satisfies the $\Delta_{2}$-condition, denoted $\Phi \in \Delta_{2}$, if there exists a constant $K>0$ and $x_{0}>0$ such that $\Phi(2 x) \leq K \Phi(x)$ whenever $x \geq x_{0}$ if $G$ is compact and the same inequality holds with $x_{0}=0$ if $G$ is non compact.

The Orlicz space, denoted $L^{\Phi}(G)$, is a vector space consisting of measurable functions, defined as

$$
L^{\Phi}(G)=\left\{f: G \rightarrow \mathbb{C}: \mathrm{f} \text { is measurable and } \int_{G} \Phi(\beta|f|) d x<\infty \text { for some } \beta>0\right\}
$$

The Orlicz space $L^{\Phi}(G)$ is a Banach space when equipped with the norm

$$
N_{\Phi}(f)=\inf \left\{k>0: \int_{G} \Phi\left(\frac{|f|}{k}\right) d x \leq 1\right\} .
$$

The above norm is called as the Luxemburg norm or Gauge norm. If $(\Phi, \Psi)$ is a complementary Young pair, then there is a norm on $L^{\Phi}(G)$, equivalent to the Luxemberg norm, given by,

$$
\|f\|_{\Phi}=\sup \left\{\int_{G}|f g| d x: \int_{G} \Psi(|g|) d x \leq 1\right\} .
$$

This norm is called as the Orlicz norm.

Let $C_{c}(G)$ denote the space of all continuous functions on $G$ with compact support. If a Young function $\Phi$ satisfies the $\Delta_{2}$-condition, then $C_{c}(G)$ is dense in $L^{\Phi}(G)$. Further, if the complementary function $\Psi$ is such that $\Psi$ is continuous and $\Psi(x)=0$ iff $x=0$, then the dual of $\left(L^{\Phi}(G), N_{\Phi}(\cdot)\right)$ is isometrically isomorphic to $\left(L^{\Psi}(G),\|\cdot\|_{\Psi}\right)$. In particular, if both $\Phi$ and $\Psi$ satisfies the $\Delta_{2}$-condition, then $L^{\Phi}(G)$ is reflexive.

For more details on Orlicz spaces, we refer the readers to [13].

Let $\Phi$ and $\Psi$ be a pair of complementary Young functions satisfying the $\Delta_{2}$ condition. Let

$A_{\Phi}(G)=\left\{u=\sum_{n=1}^{\infty} f_{n} * \check{g_{n}}:\left\{f_{n}\right\} \subset L^{\Phi}(G),\left\{g_{n}\right\} \in L^{\Psi}(G)\right.$ and $\left.\sum_{n=1}^{\infty} N_{\Phi}\left(f_{n}\right)\left\|g_{n}\right\|_{\Psi}<\infty\right\}$.

Note that if $u \in A_{\Phi}(G)$ then $u \in C_{0}(G)$. If $u \in A_{\Phi}(G)$, define $\|u\|_{A_{\Phi}}$ as

$$
\|u\|_{A_{\Phi}}:=\inf \left\{\sum_{n=1}^{\infty} N_{\Phi}\left(f_{n}\right)\left\|g_{n}\right\|_{\Psi}: u=\sum_{n=1}^{\infty} f_{n} * \check{g_{n}}\right\} .
$$

The space $A_{\Phi}(G)$ equipped with the above norm and with the pointwise addition and multiplication becomes a commutative Banach algebra [14, Theorem 3.4]. In fact, $A_{\Phi}(G)$ is a commutative, regular and semisimple banach algebra with spectrum homeomorphic to $G$ [14, Corollary 3.8]. This Banach algebra $A_{\Phi}(G)$ is called as the Orlicz Figà-Talamanca Herz algebra. Let

$$
B_{\Phi}(G):=\left\{u \in C(G): u v \in A_{\Phi}(G) \forall v \in A_{\Phi}(G)\right\}
$$


equipped with the norm $\|u\|_{B_{\Phi}}=\sup \left\{\|u v\|_{A_{\Phi}}: v \in A_{\Phi}(G),\|v\|_{A_{\Phi}}=1\right\}$. Then, with the above norm, $B_{\Phi}(G)$ becomes a commutative Banach algebra with pointwise addition and multiplication.

Let $\mathcal{B}\left(L^{\Phi}(G)\right)$ be the linear space of all bounded linear operators on $L^{\Phi}(G)$ equipped with the operator norm. For a bounded complex Radon measure $\mu$ on $G$ and $f \in L^{\Phi}(G)$, define $T_{\mu}: L^{\Phi}(G) \rightarrow L^{\Phi}(G)$ by $T_{\mu}(f)=\mu * f$. It is clear that $T_{\mu} \in \mathcal{B}\left(L^{\Phi}(G)\right)$. Let $P M_{\Phi}(G)$ denote the closure of

$$
\left\{T_{\mu}: \mu \text { is a bounded complex Radon measure }\right\}
$$

in $\mathcal{B}\left(L^{\Phi}(G)\right)$ with respect to the ultraweak topology. It is proved in [14, Theorem 3.5], that for a locally compact group $G$, the dual of $A_{\Phi}(G)$ is isometrically isomorphic to $P M_{\Psi}(G)$. By [14, Theorem 3.6] singletons are sets of spectral synthesis for $A_{\Phi}(G)$. Further, every closed subgroup is a set of local synthesis for $A_{\Phi}(G)$.

Throughout this paper, $G$ will denote a locally compact group with a fixed left Haar measure $d x$. Further $\Phi$ will always denote a Young function whose complementary Young function is $\Psi$ and the pair $(\Phi, \Psi)$ satisfies the $\Delta_{2}$-condition.

\section{Non-DEgenerate $*$-REPRESEntations of $A_{\Phi}(G)$}

In this section, motivated by the results of $[10,11]$, we describe all the non-degenerate *-representations of $A_{\Phi}(G)$. Throughout this section and the next, $\mathcal{H}$ will denote a Hilbert space.

Proposition 3.1. Let $\mu$ be a bounded positive Radon measure on $G$.

(i) For each $u \in A_{\Phi}(G)$, the mapping $\pi_{\mu}(u): f \mapsto u f$ is a bounded linear operator on $L^{2}(G, d \mu)$.

(ii) The mapping $u \mapsto \pi_{\mu}(u)$ defines a $*$-representation of $A_{\Phi}(G)$ on $\mathcal{B}\left(L^{2}(G, d \mu)\right)$.

(iii) If $\mu$ is bounded, then $\pi_{\mu}$ is a cyclic representation of $A_{\Phi}(G)$ with the constant 1 function as cyclic vector.

Proof. (i) and (ii) are just a routine check.

(iii) We show that the constant 1 function is a cyclic vector. Since the measure $\mu$ is finite, the conclusion follows from the density of $A_{\Phi}(G) \cap C_{c}(G)$ in $C_{c}(G)$ with respect to the $L^{2}(G, d \mu)$-norm.

Corollary 3.2. If $\mu$ is a positive Radon measure on $G$ (not necessarily bounded) then $\pi_{\mu}$ (defined as in Proposition 3.1) is non-degenerate.

Proof. Let $\mu$ be a positive Radon measure on $G$. By [3, Pg. 33, 2.2.7], it is enough to show that the representation $\pi_{\mu}$ is a direct sum of cyclic representations. By [1, INT IV.77] and [1, INT V.14, Proposition 4], it follows that

$$
L^{2}(G, d \mu) \cong \underset{\alpha \in \wedge}{\oplus} L^{2}\left(G, d \mu_{\alpha}\right),
$$

where $\left\{\mu_{\alpha}\right\}_{\alpha \in \wedge}$ is a summable family of measures with pairwise disjoint support. Now the conclusion follows from (iii) of Proposition 3.1.

In the next result, we characterise all cyclic $*$-representations.

Theorem 3.3. Let $\{\pi, \mathcal{H}\}$ be a cyclic $*$-representation of $A_{\Phi}(G)$. Then there exists a bounded positive Radon measure $\mu$ such that $\pi$ is unitarily equivalent to the representation $\left\{\pi_{\mu}, L^{2}(G, d \mu)\right\}$ given in Proposition 3.1.

Proof. Let $u \in A_{\Phi}(G)$. Then, by [15, Pg. 22], it follows that $\|\pi(u)\|_{s p} \leq\|u\|_{s p}$. By [14, Theorem 3.4], $A_{\Phi}(G)$ is a commutative Banach algebra and hence the spectral norm and 
the operator norm for $\pi(u)$ coincides. Further, as $A_{\Phi}(G)$ is semi-simple and the fact that the spectrum of $A_{\Phi}(G)$ is $G$ [14, Corollary 3.81], $\|u\|_{s p}=\|u\|_{\infty}$. Thus,

$$
\|\pi(u)\|_{\mathcal{B}(\mathcal{H})} \leq\|u\|_{\infty} .
$$

As a consequence of this inequality and the fact that $A_{\Phi}(G)$ is dense in $C_{0}(G)$, it follows that $\pi$ extends to a $*$-representation of $C_{0}(G)$ on $\mathcal{H}$, still denoted as $\pi$. Note that $\pi$ is a cyclic *-representation of the $C^{*}$-algebra $C_{0}(G)$. Let $\varphi$ be the cyclic vector of the representation $\left\{\pi, C_{0}(G)\right\}$. Define $T_{\varphi}: C_{0}(G) \rightarrow \mathbb{C}$ as

$$
T_{\varphi}(u)=\langle\pi(u) \varphi, \varphi\rangle, u \in C_{0}(G) .
$$

It is clear that $T_{\varphi}$ is a positive linear functional on $C_{0}(G)$ and hence, by Riesz representation theorem, there exists a bounded positive Radon measure $\mu$ such that

$$
T_{\varphi}(u)=\int_{G} u d \mu .
$$

Let $\pi_{\mu}$ denote the cyclic $*$-representation of $A_{\Phi}(G)$ on $L^{2}(G, d \mu)$, given by Proposition 3.1 .

We now claim that the representations $\pi$ and $\pi_{\mu}$ of $A_{\Phi}(G)$ are unitarily equivalent. Since $\varphi$ is a cyclic vector, in order to prove the above claim, it is enough to show that the correspondence $\pi(u) \varphi \mapsto u .1$ is an isometry and commutes with $\pi$ and $\pi_{\mu}$. Note that the above correspondence is well-defined by (3.1). Let $T$ denote the above well-defined correspondence.

We now show that $T$ is an isometry. Let $u \in A_{\Phi}(G)$. Then

$$
\begin{aligned}
\langle\pi(u) \varphi, \pi(u) \varphi\rangle & =\left\langle\pi^{*}(u) \pi(u) \varphi, \varphi\right\rangle \\
& =\langle\pi(\bar{u} u) \varphi, \varphi\rangle(\pi \text { is a } * \text {-homomorphism }) \\
& =\int_{G}|u|^{2} d \mu=\langle\varphi, \varphi\rangle .
\end{aligned}
$$

Finally, we show that $T$ intertwines with $\pi$ and $\pi_{\mu}$. Let $u \in A_{\Phi}(G)$. Then, for $v \in A_{\phi}(G)$, we have,

$$
\begin{aligned}
T(\pi(u)(\pi(v) \varphi)) & =T((\pi(u) \pi(v)) \varphi) \\
& =T(\pi(u v) \varphi)=u v .1 \\
& =\pi_{\mu}(u)(v \cdot 1)=\pi_{\mu}(u)(T(\pi(v) \varphi)) .
\end{aligned}
$$

Here is the main result of this section, describing all the non-degenerate Hilbert space representations of $A_{\Phi}(G)$.

Corollary 3.4. If $\{\pi, \mathcal{H}\}$ is any non-degenerate $*$-representation of $A_{\Phi}(G)$ then $\pi$ is unitarily equivalent to $\left\{\pi_{\mu}, L^{2}(G, d \mu)\right\}$ for some positive measure $\mu$.

Proof. Let $\{\pi, \mathcal{H}\}$ be a non-degenerate $*$-representation of $A_{\Phi}(G)$. By [3, Proposition 2.2.7], $\pi$ is a direct sum of cyclic $*$-representations $\left\{\pi_{\alpha}, \mathcal{H}_{\alpha}\right\}_{\alpha \in \wedge}$. For each $\alpha \in \wedge$, by Theorem 3.3, there exists a bounded positive measure $\mu_{\alpha}$ such that the representations $\left\{\pi_{\alpha}, \mathcal{H}_{\alpha}\right\}$ and $\left\{\pi_{\mu_{\alpha}}, L^{2}\left(G, d \mu_{\alpha}\right)\right\}$ are unitarily equivalent.

Suppose that the family $\left\{\mu_{\alpha}\right\}_{\alpha \in \wedge}$ is summable. Let $\mu=\sum_{\alpha \in \wedge} \mu_{\alpha}$. Then $\mu$ will be a positive measure and

$$
\left\{\pi_{\mu}, L^{2}(G, d \mu)\right\} \cong \underset{\alpha \in \wedge}{\oplus}\left\{\pi_{\mu_{\alpha}}, L^{2}\left(G, d \mu_{\alpha}\right)\right\} \cong \underset{\alpha \in \wedge}{\oplus}\left\{\pi_{\alpha}, \mathcal{H}_{\alpha}\right\} \cong\{\pi, \mathcal{H}\}
$$


Thus, we are done if we can show that $\left\{\mu_{\alpha}\right\}_{\alpha \in \wedge}$ is a summable family. Let $f: G \rightarrow \mathbb{C}$ be a continuous function with compact support. Then $\underset{\alpha \in \wedge}{\oplus} f \in \underset{\alpha \in \wedge}{\oplus} L^{2}\left(G, \mu_{\alpha}\right)$ and hence,

$$
\sum_{\alpha \in \wedge}\left(\int_{G}|f|^{2} d \mu_{\alpha}\right)^{1 / 2}<\infty .
$$

Now,

$$
\begin{aligned}
\sum_{\alpha \in \wedge}\left|\mu_{\alpha}(f)\right| & =\sum_{\alpha \in \wedge}\left|\int_{G} f d \mu_{\alpha}\right| \leq \sum_{\alpha \in \wedge} \int_{G}|f| d \mu_{\alpha} \\
& \leq \sum_{\alpha \in \wedge}\left(\int_{G}|f|^{2} d \mu_{\alpha}\right)^{1 / 2}\left(\int_{G}|1|^{2} d \mu_{\alpha}\right)^{1 / 2} \\
& =\sum_{\alpha \in \wedge}\left(\int_{G}|f|^{2} d \mu_{\alpha}\right)^{1 / 2}\left(\mu_{\alpha}(G)\right)^{1 / 2} \\
& \leq \sup _{\alpha \in \wedge}\left(\mu_{\alpha}(G)\right)^{1 / 2} \sum_{\alpha \in \wedge}\left(\int_{G}|f|^{2} d \mu_{\alpha}\right)^{1 / 2} \\
& \leq\left(\sup _{\alpha \in \wedge} \mu_{\alpha}(G)\right)^{1 / 2} \sum_{\alpha \in \wedge}\left(\int_{G}|f|^{2} d \mu_{\alpha}\right)^{1 / 2}<\infty .
\end{aligned}
$$

The boundedness of $\sup _{\alpha \in \wedge} \mu_{\alpha}(G)$ follows from the uniform boundedness principle and from $(3.2)$.

\section{Non-DEgEnerate $*$-REPRESEntations of $B_{\Phi}(G)$}

In this section, we show that the non-degenerate representations described in the previous section can be extended uniquely to $B_{\Phi}(G)$.

Theorem 4.1. Let $\{\pi, \mathcal{H}\}$ be a non-degenerate $*$-representation of $A_{\Phi}(G)$.

(i) For each $u \in B_{\Phi}(G)$, there exists a unique operator $\widetilde{\pi}(u) \in \mathcal{B}(\mathcal{H})$ such that, $\forall v \in$ $A_{\Phi}(G)$

$$
\tilde{\pi}(u) \pi(v)=\pi(u v)
$$

and

$$
\tilde{\pi}(v)=\pi(v) .
$$

(ii) The mapping $u \mapsto \widetilde{\pi}(u)$ defines a non-degenerate $*$-representation of $B_{\Phi}(G)$ on $\mathcal{H}$.

Proof. (i) Let $\pi$ be a non-degenerate $*$-representation of $A_{\Phi}(G)$. By [3, Proposition 2.2.7], $\pi$ is a direct sum of cyclic $*$-representations, say $\left\{\pi_{\alpha}, \mathcal{H}_{\alpha}\right\}_{\alpha \in \wedge}$. If we can prove (i) for each of these $\pi_{\alpha}$ 's, then the argument for $\pi$ is similar to the one given in Corollary 3.4. Thus, in order to prove this, we assume that the representation $\pi$ is cyclic. Since $\pi$ is a cyclic $*$-representation, by Theorem $3.3, \pi$ is unitarily equivalent to $\pi_{\mu}$, for some bounded positive Radon measure $\mu$. So, without loss of generality, let us assume that the non-degenerate $*$-representation of $A_{\Phi}(G)$ is $\pi_{\mu}$ for some bounded positive Radon measure $\mu$.

Let $u \in B_{\Phi}(G)$. By Proposition 3.1, the space $\mathcal{K}:=\operatorname{span}\left\{\pi_{\mu}(v) .1: v \in A_{\Phi}(G)\right\}$ is dense in $L^{2}(G, d \mu)$. Define $\widetilde{\pi_{\mu}}(u): \mathcal{K} \rightarrow L^{2}(G, d \mu)$ as

$$
\widetilde{\pi_{\mu}}(u)\left(\pi_{\mu}(v) \cdot 1\right)=\pi_{\mu}(u v) \cdot 1 \text {. }
$$


It is clear that $\widetilde{\pi_{\mu}}(u)$ is linear. We now claim that $\widetilde{\pi_{\mu}}(u)$ is bounded. Let $v \in A_{\Phi}(G)$. Then

$$
\begin{aligned}
\left\|\widetilde{\pi_{\mu}}(u)\left(\pi_{\mu}(v) \cdot 1\right)\right\|_{2}^{2} & =\left\|\pi_{\mu}(u v) \cdot 1\right\|_{2}^{2} \\
& =\int_{G}\left|\pi_{\mu}(u v) \cdot 1\right|^{2} d \mu \\
& =\int_{G}|u v|^{2} d \mu \\
& \leq\|u\|_{\infty}^{2} \int_{G}|v|^{2} d \mu \leq\|u\|_{B_{\Phi}}^{2}\left\|\pi_{\mu}(v) \cdot 1\right\|_{2}^{2} .
\end{aligned}
$$

Thus, $\widetilde{\pi_{\mu}}(u)$ extends to a bounded linear operator on $L^{2}(G, d \mu)$, still denoted $\widetilde{\pi_{\mu}}(u)$. Further, it is clear that, for $u \in B_{\Phi}(G)$ and $v \in A_{\Phi}(G), \widetilde{\pi_{\mu}}(u) \pi_{\mu}(v)=\pi_{\mu}(u v)$. Now, let $v \in A_{\Phi}(G)$. Then, for $u \in A_{\Phi}(G)$,

$$
\widetilde{\pi_{\mu}}(v)\left(\pi_{\mu}(u) \cdot 1\right)=\pi_{\mu}(v u) \cdot 1=\pi_{\mu}(v)\left(\pi_{\mu}(u) \cdot 1\right) .
$$

Again, as $\mathcal{K}$ is dense in $L^{2}(G, d \mu)$, it follows that $\widetilde{\pi_{\mu}}(v)=\pi_{\mu}(v)$ for all $v \in A_{\Phi}(G)$.

Finally, uniqueness follows from condition (4.1).

(ii) Non-degeneracy of $\widetilde{\pi}$ follows from the fact that $\pi$ is non-degenerate. Further, homomorphism property of $\widetilde{\pi}$ follows from (4.1). Now, we show that $\widetilde{\pi}$ preserves involution. Let $u \in B_{\Phi}(G)$. Then, for $v \in A_{\Phi}(G)$ and $\xi, \eta \in \mathcal{H}$, we have

$$
\begin{aligned}
\left\langle\widetilde{\pi}(u)^{*} \pi(v) \xi, \eta\right\rangle & =\langle\xi, \pi(\bar{v}) \widetilde{\pi}(u) \eta\rangle \\
& =\langle\xi, \widetilde{\pi}(\bar{v}) \widetilde{\pi}(u) \eta\rangle \text { by }(4.2)) \\
& =\langle\xi, \widetilde{\pi}(u \bar{v}) \eta\rangle(\widetilde{\pi} \text { is a homomorphism) } \\
& =\langle\xi, \pi(u \bar{v}) \eta\rangle \text { (by (4.2)) } \\
& =\left\langle\xi, \pi(\bar{u} \bar{v})^{*} \eta\right\rangle(\pi \text { preserves involution) } \\
& =\langle\pi(\bar{u} v) \xi, \eta\rangle \\
& =\langle\widetilde{\pi}(\bar{u}) \pi(v) \xi, \eta\rangle .(\text { by }(4.1))
\end{aligned}
$$

Since the representation $\pi$ is non-degenerate, the space $\left\{\pi(u) \xi: u \in A_{\Phi}(G), \xi \in \mathcal{H}\right\}$ is dense in $\mathcal{H}$. Thus, it follows that $\widetilde{\pi}(u)^{*}=\widetilde{\pi}(\bar{u})$ for all $u \in B_{\Phi}(G)$.

The following corollary is the converse of the above theorem.

Corollary 4.2. Let $\{\pi, \mathcal{H}\}$ be a *-representation of $B_{\Phi}(G)$ such that $\left.\pi\right|_{A_{\Phi}}$ is nondegenerate. Then, $\widetilde{\left.\pi\right|_{A_{\Phi}}}=\pi$ and $\pi$ is non-degenerate.

Proof. Let $u \in B_{\Phi}(G)$ and $v \in A_{\Phi}(G)$. Then

$$
\left.\pi(u) \pi\right|_{A_{\Phi}}(v)=\pi(u) \pi(v)=\pi(u v)=\left.\pi\right|_{A_{\Phi}}(u v) .
$$

Thus, by Theorem 4.1, it follows that $\widetilde{\left.\pi\right|_{A_{\Phi}}}=\pi$. Again by Theorem 4.1, $\widetilde{\left.\pi\right|_{A_{\Phi}}}$ is nondegenerate and hence it follows that the representation $\pi$ is non-degenerate.

\section{Application to ergodic sequences in $A_{\Phi}(G)$}

In this section, we discuss an application of ergodic sequences. This section is also motivated from [10] and [11].

Let

$$
\begin{aligned}
& S_{B}^{\Phi}=\left\{u \in B_{\Phi}(G):\|u\|_{B_{\Phi}}=u(e)=1\right\} \\
& S_{A}^{\Phi}=\left\{u \in A_{\Phi}(G):\|u\|_{A_{\Phi}}=u(e)=1\right\} .
\end{aligned}
$$

Before we proceed to the main result of this section, here we give an appropriate definition. 
Definition 5.1. A sequence $\left\{u_{n}\right\} \subset S_{B}^{\Phi}$ is said to be strongly (resp. weakly) ergodic if for any non-degenerate $*$-representation $\left\{\pi, \mathcal{H}_{\pi}\right\}$ of $A_{\Phi}(G)$ the sequence $\left\{\tilde{\pi}\left(u_{n}\right) \eta\right\}$ converges strongly (resp. weakly) to an element of $\mathcal{H}_{f}$, for every $\eta \in \mathcal{H}$, where

$$
\mathcal{H}_{f}=\left\{\xi \in \mathcal{H}: \pi(u) \xi=\xi \forall u \in S_{A}^{\Phi}\right\} .
$$

Our next theorem is the main result of this section.

Theorem 5.2. For a sequence $\left\{u_{n}\right\}$ in $S_{B}^{\Phi}$, the following statements are equivalent:

(i) the sequence $\left\{u_{n}\right\}$ is strongly ergodic.

(ii) the sequence $\left\{u_{n}\right\}$ is weakly ergodic.

(iii) the sequence $\left\{u_{n}(x)\right\}$ converges to 0 for every $x \in G$ with $x \neq e$.

Proof. (i) $\Rightarrow$ (ii) is obvious.

(ii) $\Rightarrow$ (iii). Fix $x \in G$ with $x \neq e$. Define $\pi: A_{\Phi}(G) \rightarrow \mathbb{C}$ as $\pi(u)=u(x)$. Then $\pi$ defines a non-degenerate $*$-representation of $A_{\Phi}(G)$ on $\mathbb{C}$. By Theorem 4.1, the representation $\{\pi, \mathbb{C}\}$ can be extended uniquely to a non-degenerate $*$-representation $\widetilde{\pi}$ of $B_{\Phi}(G)$ on $\mathbb{C}$ such that $\widetilde{\pi}(u) z=u(x) z$ for all $u \in B_{\Phi}(G)$. Since $\left\{u_{n}\right\}$ is weakly ergodic the set $\left\{\mathbb{C}_{f}\right\}$ is non-empty. In order to prove (iii) it is enough to show that the set $\mathbb{C}_{f}$ consists only of the zero vector. Suppose to the contrary that there exists $0 \neq z \in \mathbb{C}_{f}$. Since $G$ is Hausdorff, there exists an open set $U$ containing $e$ but not $x$. Let $v$ denote the function given by [14, Proposition 5.5], corresponding the open set $U$. Then $v \in S_{A}^{\Phi}$ and $v(x) z=0$, which is a contradiction. Thus the set $\mathbb{C}_{f}$ consists only of the zero vector. Hence (iii).

(iii) $\Rightarrow$ (i). Let $\pi$ be a non-degenerate $*$-representation of $A_{\Phi}(G)$. By Corollary 3.4, $\pi$ is unitarily equivalent to the representation $\left\{\pi_{\mu}, L^{2}(G, d \mu)\right\}$ for some positive measure $\mu$ defined on $G$. So, without loss of generality, let us assume that $\pi$ is of the form $\pi_{\mu}$ for some positive measure $\mu$ on $G$. Let $\widetilde{\pi_{\mu}}$ denote the extension of $\pi_{\mu}$ from $A_{\Phi}(G)$ to $B_{\Phi}(G)$ as in Theorem 4.1. Let $f \in L^{2}(G, d \mu)$. We now claim that the sequence $\left\{\widetilde{\pi_{\mu}}\left(u_{n}\right)(f)\right\}$ converges strongly. As $L^{2}(G, d \mu)$ is complete, in order to prove the claim, it is enough to show that the sequence $\left\{\widetilde{\pi_{\mu}}\left(u_{n}\right)(f)\right\}$ is Cauchy. Note that, for any $n, m \in \mathbb{N}$,

$$
\left|u_{n}(x)-u_{m}(x)\right|^{2}|f(x)|^{2} \leq 4|f(x)|^{2} \text { a.e. }
$$

Thus, by dominated convergence theorem and by (iv), we have,

$$
\begin{aligned}
\left\|\widetilde{\pi_{\mu}}\left(u_{n}\right)(f)-\widetilde{\pi_{\mu}}\left(u_{m}\right)(f)\right\|_{2}^{2} & =\int_{G}\left|\widetilde{\pi_{\mu}}\left(u_{n}\right)(f)(x)-\widetilde{\pi_{\mu}}\left(u_{m}\right)(f)(x)\right|^{2} d \mu(x) \\
& =\int_{G}\left|u_{n}(x)-u_{m}(x)\right|^{2}|f(x)|^{2} d \mu(x) \\
& \rightarrow 0 .
\end{aligned}
$$

Let $g \in L^{2}(G, d \mu)$ denote the limit of the sequence $\left\{\widetilde{\pi_{\mu}}\left(u_{n}\right)(f)\right\}$. Our next claim is that $g$ is a fixed point of $\pi_{\mu}(u)$ for each $u \in S_{A}^{\Phi}$. Again, this is a consequence of the dominated convergence theorem.

\section{Spectral subspaces}

In this section, we study the spectral subspaces associated to a non-degenerate Banach space representation of $A_{\Phi}(G)$. Our main aim in this section is to prove Corollary 6.9. Most of the ideas of this section are taken from [12].

Definition 6.1. Let $T \in P M_{\Psi}(G)$. Then the support of $T$ is defined as

$$
\operatorname{supp}(T)=\left\{x \in G: u \in A_{\Phi}(G), u(x) \neq 0 \Rightarrow u . T \neq 0\right\} .
$$

Here we recall some of the properties of the support of $T$ in the form of a Lemma [7, Pg. 101]. 


\section{Lemma 6.2.}

(i) If $T_{1}, T_{2} \in P M_{\Psi}(G)$ then $\operatorname{supp}\left(T_{1}+T_{2}\right) \subseteq \operatorname{supp}\left(T_{1}\right) \cup \operatorname{supp}\left(T_{2}\right)$.

(ii) If $u \in A_{\Phi}(G)$ and $T \in P M_{\Psi}(G)$ then $\operatorname{supp}(u . T) \subseteq \operatorname{supp}(u) \cap \operatorname{supp}(T)$.

(iii) If $c \in \mathbb{C}$ and $T \in P M_{\Psi}(G)$ then $\operatorname{supp}(c T) \subseteq \operatorname{supp}(T)$.

(iv) Let $T \in P M_{\Psi}(G)$ and let $E$ be a closed subset of $G$. If a net $\left\{T_{\alpha}\right\} \subset P M_{\Psi}(G)$ converges weakly to $T$ with $\operatorname{supp}\left(T_{\alpha}\right) \subset E$ for all $\alpha$, then $\operatorname{supp}(T) \subset E$.

Let $X$ be a Banach space and let $\pi$ be an algebra representation of $A_{\Phi}(G)$ on $X$. For $\varphi \in X$ and $x^{*} \in X^{*}$, define $T_{x^{*}, \varphi}: A_{\Phi}(G) \rightarrow \mathbb{C}$ as

$$
\left\langle u, T_{x^{*}, \varphi}\right\rangle:=\left\langle\pi(u) \varphi, x^{*}\right\rangle \forall u \in A_{\Phi}(G) .
$$

We say that the representation $\pi$ is continuous if $T_{x^{*}, \varphi}$ is a continuous linear functional on $A_{\Phi}(G)$ for each $\varphi \in X$ and $x^{*} \in X^{*}$. It follows from uniform boundedness principle that the linear map $\pi: A_{\Phi}(G) \rightarrow \mathcal{B}(X)$ is norm continuous.

From now onwards, $X$ will denote a Banach space and $\pi$ an algebra representation of $A_{\Phi}(G)$ on $X$.

Let $E$ be a closed subset of $G$. Define

$$
X_{E}:=\left\{\varphi \in X: \operatorname{supp}\left(T_{x^{*}, \varphi}\right) \subseteq E \forall x^{*} \in X^{*}\right\} .
$$

Remark 6.3. An immediate consequence of the above definition is that, if $E=G$ then $X_{E}=X$.

Lemma 6.4. The set $X_{E}$ is a closed $\pi$-invariant subspace of $X$.

Proof. Note that for any $x^{*} \in X^{*}, \varphi_{1}, \varphi_{2} \in X_{E}$ and $\alpha \in \mathbb{C}$, we have

$$
T_{x^{*}, \varphi_{1}+\alpha \varphi_{2}}=T_{x^{*}, \varphi_{1}}+\alpha T_{x^{*}, \varphi_{2}} .
$$

Thus, it follows from (i) and (iii) of Lemma 6.2 that $X_{E}$ is a linear space. Further, closedness of $X_{E}$ is an immediate consequence of (iv) from Lemma 6.2. Again, note that, for any $u \in A_{\Phi}(G), \varphi \in X$ and $x^{*} \in X^{*}$, we have $T_{x^{*}, \pi(u) \varphi}=u . T_{x^{*}, \varphi}$ and hence the invariance of $X_{E}$ under $\pi$ follows from (ii) of Lemma 6.2.

The subspace $X_{E}$ is called as the spectral subspace associated with the representation $\pi$ and the closed set $E$.

Lemma 6.5. Let $\pi$ be a non-degenerate representation of $A_{\Phi}(G)$.

(i) The space $X_{\emptyset}=\{0\}$.

(ii) If $\left\{E_{i}\right\}$ is an arbitrary collection of closed subsets of $G$, then $X_{i} E_{i}=\bigcap_{i} X_{E_{i}}$.

Proof. (i) is an easy consequence of the non-degeneracy of $\pi$, while (ii) is trivial.

The following is an immediate corollary of Remark 6.3 and Lemma 6.5.

Corollary 6.6. There exists a smallest closed non-empty set $E$ of $G$ such that $X_{E}=X$.

Proposition 6.7. Let $K_{1}$ and $K_{2}$ be disjoint compact subsets of $G$. Then $X_{K_{1} \cup K_{2}}=$ $X_{K_{1}} \oplus X_{K_{2}}$.

Proof. The proof of this follows exactly as given in [12, Proposition 2 (iii)].

Theorem 6.8. Let $\pi$ be a non-degenerate representation of $A_{\Phi}(G)$ such that the only spectral subspaces are the trivial subspaces. Then there exists $x \in G$ such that $X_{\{x\}}=X$.

Proof. Choose a smallest non-empty closed set $E$ such that $X_{E}=X$, which is possible by Corollary 6.6. Suppose there exists $x, y \in E$ such that $x \neq y$. As $G$ is locally compact and Hausdorff, there exists an open set $U$ and a compact set $K$ such that $x \in U \subset K$ and $y \notin K$. Since $A_{\Phi}(G)$ is regular, there exists $u \in A_{\Phi}(G)$ such that $u=1$ on $U$ and $\operatorname{supp}(u) \subset K$. 
Let $v \in A_{\Phi}(G)$ be arbitrary. Let $v_{1}=v-u v$ and $v_{2}=u v$ so that $v=v_{1}+v_{2}$. Let $V=\left\{z \in G: v_{1}(z) \neq 0\right\}$. The choice of $u$ tells us that $x \notin \bar{V}$. Again, using the regularity of $A_{\Phi}(G)$, choose a function $w \in A_{\Phi}(G)$ such that $w=1$ on some open set $W$ containing $x$ and $\operatorname{supp}(w) \cap V=\emptyset$. Further, it is clear that $v_{1} w=0$.

We now claim that $\pi(v)=0$. Let $\varphi \in X$ and $x^{*} \in X^{*}$. If $z \in W$, then $w(z)=1$ and hence $T_{x^{*}, \pi\left(v_{1} w\right) \varphi}=0$ as $T_{x^{*}, \pi\left(v_{1} w\right) \varphi}=w \cdot T_{x^{*}, \pi\left(v_{1}\right) \varphi}$. Thus $\operatorname{supp}\left(T_{x^{*}, \pi\left(v_{1}\right) \varphi}\right) \subset W^{c}$. Therefore, using the non-degeneracy of $\pi$, it follows that, if $\pi\left(v_{1}\right) \varphi \neq 0$ then $X_{W^{c}}=X$ and hence, by the choice of the set $E$, it follows that $E$ is a subset of $W^{c}$. On the other hand, $x \notin W^{c}$ and $x \in E$ and hence $E$ is not a subset of $W^{c}$. Therefore, $\pi\left(v_{1}\right)=0$. Similarly, one can show that $\pi\left(v_{2}\right)=0$. Thus $\pi(v)=0$. Since $v$ is arbitrary, it follows that $\pi(v)=0$ for all $v \in A_{\Phi}(G)$, which is a contradiction. Thus the set $E$ is a singleton.

Corollary 6.9. Let $\pi$ be a non-degenerate representation of $A_{\Phi}(G)$ such that the only spectral subspaces are the trivial subspaces. Then $\pi$ is a character.

Proof. By Theorem 6.8, there exists $x \in G$ such that $X_{\{x\}}=X$, i.e.,

$$
\operatorname{supp}\left(T_{x^{*}, \varphi}\right) \subset\{x\}
$$

for all $\varphi \in X$ and $x^{*} \in X^{*}$. As singletons are sets of spectral synthesis for $A_{\Phi}(G)[14$, Theorem 3.6 (i)], it follows that

$$
T_{x^{*}, \varphi}=c \delta_{x}
$$

for some $c \in \mathbb{C}$. Let $u \in A_{\Phi}(G)$ such that $u(x)=1$. Then

$$
c=c\left\langle u, \delta_{x}\right\rangle=\left\langle u, c \delta_{x}\right\rangle=\left\langle u, T_{x^{*}, \varphi}\right\rangle=\left\langle\pi(u) \varphi, x^{*}\right\rangle .
$$

We now claim that $\pi$ is a character. Let $v \in A_{\Phi}(G)$. Then, for $\varphi \in X$ and $x^{*} \in X^{*}$, we have

$$
\begin{aligned}
\left\langle\pi(v) \varphi, x^{*}\right\rangle & =\left\langle v, T_{x^{*}, \varphi}\right\rangle=\left\langle v, c \delta_{x}\right\rangle(\text { by }(6.1)) \\
& =c\left\langle v, \delta_{x}\right\rangle=\left\langle\pi(u) \varphi, x^{*}\right\rangle\left\langle v, \delta_{x}\right\rangle(\text { by }(6.2)) \\
& =v(x)\left\langle\pi(u) \varphi, x^{*}\right\rangle=\left\langle v(x) \pi(u) \varphi, x^{*}\right\rangle .
\end{aligned}
$$

Since $\varphi$ and $x^{*}$ are arbitrary, it follows that $\pi(v)=u(x) \pi(u)$. Now

$$
\pi(u)=u(x) \pi(u)=u^{2}(x) \pi(u)=\pi\left(u^{2}\right)=\pi(u)^{2},
$$

i.e., $\pi(u)$ is a projection. As $\pi$ is non-degenerate, it follows that $\pi(u)$ is the identity operator $I$ on $X$. Thus

i.e., $\pi$ is a character.

$$
\pi(v)=v(x) I \forall v \in A_{\Phi}(G)
$$

\section{ACKNOWLEDGEMENT}

The first author would like to thank the University Grants Commission, India, for research grant.

\section{REFERENCES}

1. N. Bourbaki, Elements of Mathematics, Integration I, Chapters 1-6, Springer-Verlag, BerlinHeidelberg, 2004.

2. A.Derighetti, Convolution Operators on Groups, Lecture Notes Un. Mat. Ital.11, Springer, Heidelberg, 2011.

3. J. Dixmier, $C^{*}$ - algebras, North Holland, 1977.

4. P. Eymard, L'algèbre de Fourier d'un groupe localement compact, Bull. Soc. Math. France, 92 (1964) 181-236.

5. G. B. Folland, A course in abstract harmonic analysis, CRC Press, 1995.

6. R. Godement, Théorèmes taubériens et théorie spectrale, Ann. Sci. École Norm. Sup., 64 (1947), 119-138 
7. C.Herz, Harmonic synthesis for subgroups, Ann. Inst.Fourier (Grenoble) 23 (1973), no.3, 91123.

8. E. Hewitt and K. A. Ross, Abstract Harmonic Analysis I, Springer, 1979.

9. E. Kaniuth and A. T. M. LAU, Fourier and Fourier-Stieltjes algebras on locally compact groups, Mathematical Surveys and Monographs, 231, American Mathematical Society, Providence, RI, 2018.

10. A. T. M. LAU and V. LoserT, Ergodic sequences in the Fourier-Stieltjes algebra and measure algebra of a locally compact group, Trans. Amer. Math. Soc. 351 (1999), no. 1, 417428.

11. S. Mathieu Guex, Ergodic theorems for certain Banach algebras associated to locally compact groups, Ph.D. Thesis, University of Alberta, Canada, 2013.

12. K. Parthasarathy and R. Prakash, Spectral subspaces for the Fourier algebra, Colloq. Math., 108 (2007), 179-182.

13. M.M.RAO and Z.D.Ren, Theory of Orlicz Spaces, Dekker, New York, 1991.

14. Rattan Lal and N. Shravan Kumar, Orlicz Figà-Talamanca Herz algebras and invariant means, Indag. Math., 30 (2019), 340-354.

15. M. TAKESAKI, Theory of Operator algebras I, Encyclopedia of Mathematics, Springer, Vol. 124, 2001.

Department of Mathematics, Indian Institute of Technology Delhi, Delhi, 110016, India

E-mail address: rattanlaltank@gmail.com

Department of Mathematics, Indian Institute of Technology Delhi, Delhi, 110016, India

E-mail address: shravankumar@maths.iitd.ac.in

Received 19.11.2019; Revised 13.02.2020. 